\title{
ATIVIDADE DE ALGUNS INSETOS EM FLORES DE Brassica napus L. EM DOURADOS-MS E A INTERAÇÃO COM FATORES CLIMÁTICOS ${ }^{1}$
}

\author{
ROSILDA MARA MUSSURY ${ }^{2}$ \\ WEDSON DESIDERIO FERNANDES ${ }^{3}$ \\ SILVANA DE PAULA QUINTÃO SCALON ${ }^{4}$
}

\begin{abstract}
RESUMO - Objetivou-se estudar a flutuação populacional, horários de visitação e a interação com fatores climáticos de insetos visitantes de flores de Brassica napus L. em diversos estádios fenológicos. O trabalho foi realizado em Dourados, MS, Brasil. Os insetos foram coletados com rede entomológica de $30 \mathrm{~cm}$ de diâmetro, a cada duas horas, no período de 7 as 17 horas. Foram coletadas as abelhas Apis mellifera, Linnaeus, 1758, Trigona sp., o vespídeo Brachygastra lecheguana (Latreille - 1824) e uma espécie de Chloropidae (Dipte-
\end{abstract}

ra), que foi a mais abundante e freqüente nas amostragens. O pico populacional da maioria dos insetos foi das 9 às 15 horas; contudo, para Trigona sp., foi entre 9 e 11 horas. A maior frequência de insetos ocorreu durante o estádio fenológico III. A análise de correlação evidenciou que a umidade relativa entre 11 horas $(\mathrm{r}=0,57)$ e 13 horas $(\mathrm{r}=0,43)$ favoreceu a ocorrência do díptero da família $\mathrm{C}$ hloropidae. Reduções nas populações de insetos do estádio III para o IV foram devidas, provavelmente, a temperaturas mais baixas, que atingiram a média de $16,1^{\circ} \mathrm{C}$.

TERMOS PARA INDEXAÇÃO: Canola, Brassica napus, insetos polinizadores.

\section{ACTIVITY OF SOME INSECTS IN Brassica napus L. FLOWERS AT DOURADOS-MS AND THE INTERACTION WITH CLIMATIC FACTORS}

\begin{abstract}
The objective of this work was to study the populational fluctuation, the preferred visiting time, and the interaction with climatic factors in several phenological stages from visitant insects in Brassica napus L. flowers. The study was accomplished in Dourados-MS, Brazil. The insects were collected with a 30 -cm wide entomologic sweep net every 2 hours from 7 h00 to 17 h00. Apis mellifera L., 1758, Trigona sp., and Brachygastra lecheguana (Latreille - 1824) were gathered in $B$. napus flowers. A dipterous from the Chloropidae family was the insect with the largest
\end{abstract}

populational density in the samples. The large occurrence of most pollinators was between $9 \mathrm{~h} 00$ and $15 \mathrm{~h} 00$, but Trigona sp. was present in larger numbers between $9 \mathrm{~h} 00$ and $11 \mathrm{~h} 00$. The largest frequency of pollinator insects occurred during stage III of flowering. The correlation analysis indicated that the relative humidity between $11 \mathrm{~h} 00(\mathrm{r}=0,57)$ and $13 \mathrm{~h} 00(\mathrm{r}=0,43)$ favored the occurrence of the dipterous from Chloropidae family. Populational reductions of pollinator insects, during stage III to stage IV of flowering, was probably due to lower temperatures, $16^{\circ} \mathrm{C}$.

INDEX TERMS: Canola, Brassica napus, pollinators insects.

\section{INTRODUÇÃO}

A canola compreende cultivares de colza (Brassica napus L.), que foi introduzida no Brasil em 1974 pela Cooperativa Tritícola de Ijuí, RS, tornando-se uma nova opção para a agricultura devido à grande produção de néctar durante o período de inverno. É utilizada também na adubação verde, e o óleo, na alimentação humana (Martin \& Nogueira Júnior, 1993).
De acordo com Mussury \& Fernandes (2000), a ocorrência da alogamia é freqüiente em condições naturais, em razão da alta população de polinizadores em determinados períodos, favorecendo o desenvolvimento de plantas com maior tamanho de frutos e maior número de sementes. Para Kevan \& Eisikowitch (1990), a presença dos polinizadores aumenta a germinação das sementes de $B$. napus de $83 \%$ para $96 \%$.

1. Parte da dissertação de Mestrado em Agronomia realizado na Universidade Federal de Mato Grosso do Sul Dourados, MS.

2. Bióloga, Professora do Centro Universitário da Grande Dourados - UNIGRAN - Rua Balbina de Matos, 2121 79824-900 - Dourados, MS.

3. Departamento de Ciências Biológicas/Campus de Dourados/UFMS. 79825-070.

4. Bolsista de Desenvolvimento Científico Regional CNPq/UFMA-DCA, Caixa Postal 533 - 79804-970 - Dourados, MS. 
Mohammed (1935), estudando a polinização em Brassica campestris L. cv. dichotoma e B. campestris cv. sarson, observou que ambas produziram mais vagens e com maior número de sementes quando polinizadas manualmente. Embora as plantas de $B$. napus sejam auto-férteis, a polinização pelo vento ou insetos é muito importante para a obtenção de uma boa produção (Williams, 1978).

Considerando a importância da cultura da canola, objetivou-se estudar a flutuação populacional, horários de visitação e a interação com fatores climáticos de insetos visitantes de suas flores, em seus estádios fenológicos.

\section{MATERIAL E MÉTODOS}

O estudo foi desenvolvido em 1995 na Fazenda São Lourenço, Rodovia MS. 162, Km 07, no município de Dourados, MS, situado a $22^{\circ} 14^{\prime} \mathrm{S}$ e $54^{\circ} 49^{\prime} \mathrm{O}$, altitude de $452 \mathrm{~m}$, em uma área experimental de 1 ha.

As amostragens dos insetos foram semanais e feitas aleatoriamente em dez pontos durante o período de floração da canola, utilizando-se rede entomológica de $30 \mathrm{~cm}$ de diâmetro. Em cada ponto, foram realizadas dez batidas em uma distância de aproximadamente oito metros ao longo das fileiras de canola. As coletas foram realizadas a cada duas horas, de 7 as 17 horas, totalizando seis amostragens. Os indivíduos capturados foram acondicionados em sacos plásticos e levados ao laboratório para posterior triagem e encaminhamento para identificação. Foi calculado o número de indivíduos amostrados durante os diferentes horários e estádios fenológicos de florescimento.

De acordo com Informations Techniques Cetiom (1985), esses estádios foram identificados pela marcação aleatória de 10 plantas, que tiveram seus botões florais, flores e frutos contados para determinação do percentual em cada estádio. Consideraram-se: Estádio I- início do florescimento com cerca de $20 \%$ de flores abertas; Estádio II- 40\% de flores abertas; Estádio III80\% de flores abertas; Estádio IV- 100\% de flores abertas; Estádio V- início da senescência floral e as primeiras silíquas com comprimento inferior a $2,5 \mathrm{~cm}$; Estádio VI- silíquas com aproximadamente $4 \mathrm{~cm}$; Estádio VIIsilíquas com comprimento superior a $6 \mathrm{~cm}$; Estádio VIII- ausência total de flores, frutos com tamanho médio de $7 \mathrm{~cm}$.

Os dados climáticos foram obtidos na Estação Agrometeorológica do Centro de Pesquisa Agropecuária do Oeste - Embrapa-CPAO de Dourados-MS, rodovia Dourados -Caarapó, Km 5.
Foi realizada a análise de correlação entre o número de insetos, horários de visitação nos estádios de florescimento e a temperatura, umidade relativa e horas de brilho solar, usando o teste de Kendall. Os dados foram transformados para $\sqrt{\mathrm{x}+0,5}$ e os coeficientes de correlação (r) foram testados pelo teste t de Student (Snedecor \& Cochran, 1989) a 5\% e $10 \%$ de probabilidade.

\section{RESULTADOS E DISCUSSÃO}

Observaram-se nas inflorescências de B. napus dípteros da família Chloropidae, o meliponíneo Trigona sp., Apis mellifera Linnaeus, 1758 (Hymenoptera: Apidae) e o vespídeo Brachygastra lecheguana (Latreille 1824) (Hymenoptera: Vespidae) como possíveis polinizadores. Knuth (1908) e Pawlikowski (1978) observaram que, dos insetos que visitam as flores de $B . n a$ pus, há predominância das abelhas sociais e solitárias, particularmente as das famílias Andrenidae, Halictidae e Megachilidae.

Geralmente, os estádios iniciais de florescimento são preferidos pelas abelhas por haver uma maior disponibilidade de néctar. Free (1970) e Butignol (1990) observaram maior ocorrência de abelhas no início do florescimento das culturas, enquanto nos períodos finais, a incidência diminui. Segundo Butignol (1990), os óvulos fecundados, ao se desenvolverem, exigem quantidades crescentes de fotossintatos, diminuindo sua disponibilidade nos nectários das flores e interferindo na produção de néctar, provocando menor atratividade às abelhas.

A maior freqüência de insetos visitantes, alimentando-se nos nectários florais, ocorreu, de um modo geral, durante o estádio III de florescimento e no período das 9 às 15 horas, provavelmente devido à maior produção de néctar nesse horário (Figura 1). Williams (1985) demonstrou que em $B$. napus a maior atividade das abelhas ocorre entre 6 h30 e 15 horas, sendo a maior parte do pólen coletado entre 8 e 10 horas.

A ocorrência da maioria dos insetos foi das 9 às 15 horas, com o pico populacional das 13 às 15 horas; contudo, Trigona sp. teve maior ocorrência das 9 às 11 horas (Figura 1). A correlação foi positiva e significativa para a temperatura, indicando que esse fator interfere nas atividades de busca de alimento por essa espécie (Tabela 1). Outro fator que pode ter influenciado o horário de visita dessa abelha refere-se à redução da população de outros insetos, uma vez que são competidores pela mesma fonte de alimento. 

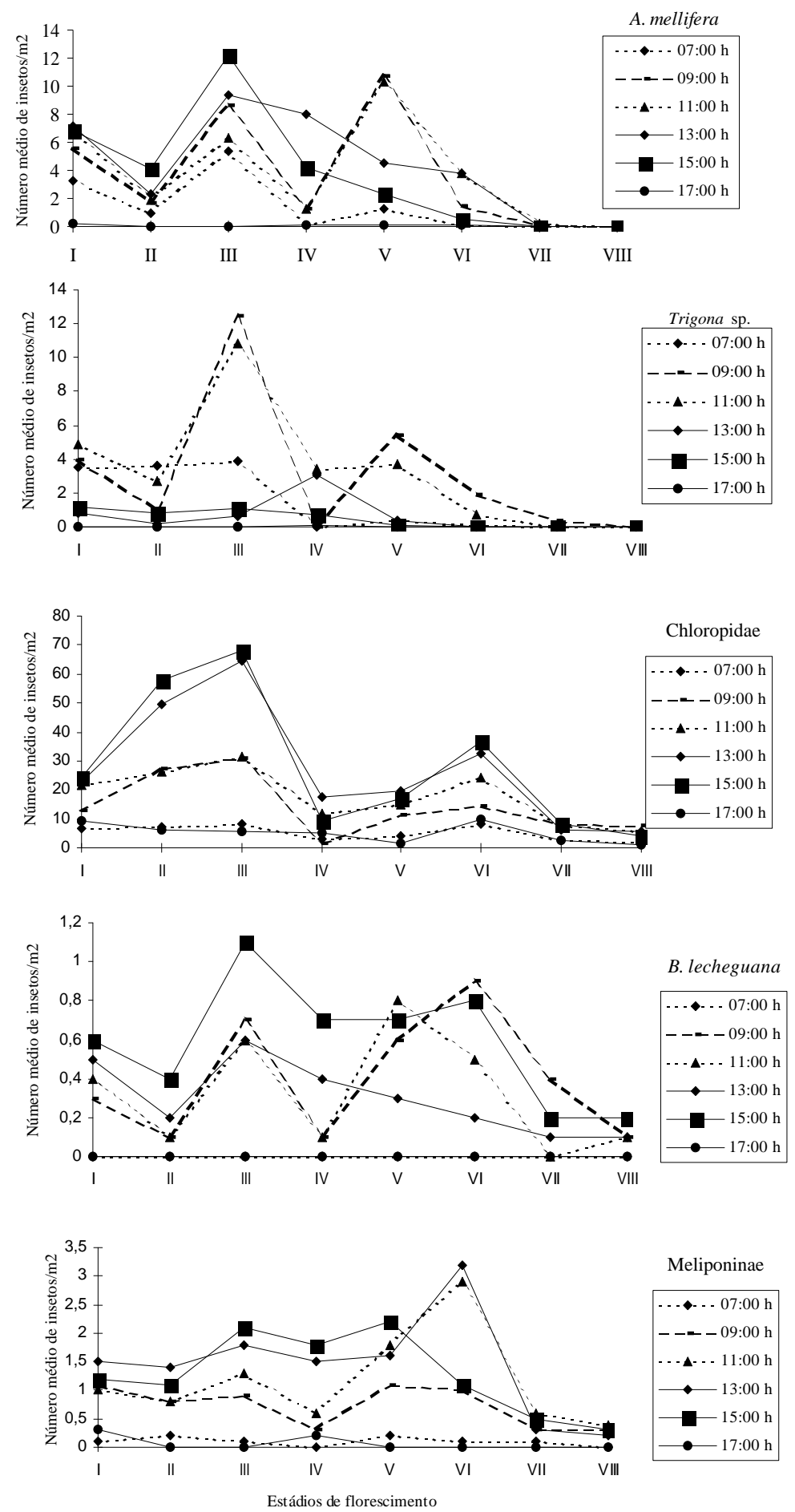

FIGURA 1 - Flutuação populacional de alguns insetos em Brassica napus L. em diferentes horários e estádios de florescimento.

Ciênc. agrotec., Lavras. V.27, n.2, p.382-388, mar./abr., 2003 
TABELA 1 - Correlação de Kendall entre os horários de amostragem e o número de insetos coletados em canola, em função da temperatura, umidade relativa e horas de brilho solar. Dourados, MS, 2001.

\begin{tabular}{|c|c|c|c|c|c|}
\hline \multicolumn{6}{|c|}{ Temperatura $\left({ }^{\circ} \mathrm{C}\right)$ / Número de insetos } \\
\hline Horário & A. mellifera & Trigona sp. & Chloropidae & B. lecheguana & Meliponinae \\
\hline $7 \mathrm{~h}$ & $0,50^{\bullet}$ & $1,00^{*}$ & $0,50^{\bullet}$ & $1,00^{\bullet}$ & $0,64^{\bullet}$ \\
\hline $9 \mathrm{~h}$ & 0,29 & $0,43^{*}$ & 0,21 & $0,79 *$ & $0,57^{*}$ \\
\hline $11 \mathrm{~h}$ & 0,07 & $0,57^{*}$ & 0,07 & $0,57^{*}$ & $0,57^{*}$ \\
\hline $13 \mathrm{~h}$ & 0,00 & $-0,21$ & $-0,07$ & 0,07 & $0,38^{\bullet}$ \\
\hline $15 \mathrm{~h}$ & $-0,07$ & $-0,21$ & $-0,07$ & 0,29 & 0,14 \\
\hline $17 \mathrm{~h}$ & $0,43^{*}$ & $0,57^{*}$ & $-0,14$ & $1,00^{\bullet}$ & $0,36^{\bullet}$ \\
\hline \multicolumn{6}{|c|}{ Umidade relativa (\%) / Número de insetos } \\
\hline Horário & A. mellifera & Trigona sp. & Chloropidae & B. lecheguana & Meliponinae \\
\hline $7 \mathrm{~h}$ & $-0,07$ & $-0,07$ & 0,14 & $1,00^{\bullet}$ & 0,29 \\
\hline $9 \mathrm{~h}$ & 0,21 & 0,00 & 0,21 & $-0,07$ & $0,43^{\bullet}$ \\
\hline $11 \mathrm{~h}$ & $0,43 *$ & 0,29 & $0,57^{*}$ & 0,14 & $0,50^{*}$ \\
\hline $13 \mathrm{~h}$ & $-0,07$ & 0,00 & $0,43^{\bullet}$ & 0,07 & 0,14 \\
\hline $15 \mathrm{~h}$ & 0,07 & 0,29 & $-0,21$ & $-0,21$ & 0,00 \\
\hline $17 \mathrm{~h}$ & $0,36^{*}$ & $0,57^{\bullet}$ & 0,29 & $1,00^{\bullet}$ & $0,43^{\bullet}$ \\
\hline
\end{tabular}

\begin{tabular}{cccccc}
\hline \multicolumn{5}{c}{ Horas de brilho solar $(\mathbf{l y}) /$ Número de insetos } \\
\hline Horário & A. mellifera & Trigona sp. & Chloropidae & B. lecheguana & Meliponinae \\
\hline $7 \mathrm{~h}$ & 0,07 & $-0,14$ & $-0,14$ & 1,00 & $-0,36^{\bullet}$ \\
$9 \mathrm{~h}$ & $-0,21$ & $-0,14$ & $-0,36$ & $-0,07$ & 0,00 \\
$11 \mathrm{~h}$ & $-0,07$ & 0,14 & $-0,21$ & 0,07 & $-0,21$ \\
$13 \mathrm{~h}$ & 0,36 & 0,50 & $-0,21$ & 0,29 & 0,00 \\
$15 \mathrm{~h}$ & 0,14 & 0,21 & $-0,21$ & 0,21 & 0,07 \\
$17 \mathrm{~h}$ & $0,57^{\bullet}$ & 1,00 & $-0,14$ & 1,00 & 0,65 \\
\hline
\end{tabular}

- $\propto=10 \% \quad * \propto=5 \%$

As abelhas Trigona sp., após pousarem nos botões florais, rasgam-no e danificam as anteras em busca de pólen. Na fase de botão, pré-antese e antese, as abelhas pousavam na corola e iniciavam o recorte das paredes com as mandíbulas. Esse recorte era feito no local de eliminação de néctar e era ampliado progressivamente, resultando numa abertura circular de tamanho variável. De acordo com as dimensões do orifício, a abelha introduzia as peças bucais ou até mesmo a cabeça e ab-

Ciênc. agrotec., Lavras. V.27, n.2, p.382-388, mar./abr., 2003 
sorvia o néctar, recuava e levantava vôo, abandonando a flor.

O comportamento de Trigona sp., nas visitas às flores de B. napus, foi descrito por Santos (1995) durante a coleta de néctar, verificando-se que a abelha corta os pedúnculos, síliquas novas e mesmo hastes tenras das inflorescências.

O comportamento forrageador e a defesa de recursos alimentares contra outras espécies de abelhas tornam Trigona sp. um visitante aparentemente desvantajoso para diversas espécies vegetais (Johnson \& Hubell, 1974). Porém Roubik \& Kerr, citados por Sazima \& Sazima (1989), discutiram as táticas de pilhagem de
Trigona e as conseqüências desse comportamento para a polinização, observando-se pólen aderido ao corpo do inseto, o qual foi considerado polinizador. No entanto, essa função de polinizador efetivo em $B$. napus precisa ser mais bem estudada, uma vez que foram observadas injúrias em botões florais e flores.

Durante o intervalo do estádio I para o II de florescimento, A. mellifera, Trigona sp. e B. lecheguana diminuíram a frequiência de visitas às flores de canola. A análise de correlação indicou que a temperatura foi o fator provável para essa redução, sendo, em média, $17,8^{\circ} \mathrm{C}$ (Tabela 2).

TABELA 2 - Correlação de Kendall entre os estádios de florescimento e o número de insetos coletados em canola, em função da temperatura e umidade relativa. Dourados, MS, 2001.

\begin{tabular}{cccccc}
\hline \multicolumn{5}{c}{ Temperatura $\left({ }^{\circ} \mathbf{C}\right) /$ Numero de insetos } \\
\hline Estádios de & A. mellifera & Trigona sp. & Chloropidae & B. lecheguana & Meliponinae \\
\hline I & 0,33 & $-0,02$ & $0,47^{\bullet}$ & $0,73^{*}$ & $0,73^{\bullet}$ \\
II & $0,47^{*}$ & $-0,60^{\bullet}$ & 0,33 & $0,60^{*}$ & 0,33 \\
III & $0,47^{*}$ & $-0,33$ & $0,60^{*}$ & $0,47^{\bullet}$ & $0,60^{*}$ \\
IV & 0,33 & 0,20 & $0,47^{\bullet}$ & $0,47^{\bullet}$ & 0,20 \\
V & $-0,20$ & $-0,33$ & $0,60^{*}$ & $0,47^{\bullet}$ & $0,47^{\bullet}$ \\
VI & 0,33 & $-0,33$ & 0,33 & 0,20 & $0,73^{*}$ \\
VII & 0,20 & $0,47^{*}$ & 0,20 & 0,33 & $-0,07$ \\
VIII & $1,0 \bullet^{\bullet}$ & $1,00^{\bullet}$ & 0,33 & $0,73^{*}$ & 0,07 \\
\hline Estádios de & A. mellifera & Trigona sp. & Chloropidae & B. lecheguana & Meliponinae \\
florescimento & 0,20 & 0,33 & $-0,33$ & $-0,47^{\bullet}$ & $-0,60^{*}$ \\
\hline I & $-0,47^{*}$ & $0,60^{\bullet}$ & $-0,20$ & $-0,33$ & $-0,33$ \\
II & $-0,33$ & $0,47^{\bullet}$ & $-0,47^{\bullet}$ & $-0,07$ & $-0,47^{\bullet}$ \\
III & $-0,20$ & $-0,20$ & $-0,60^{*}$ & $-0,33$ & 0,33 \\
IV & 0,20 & 0,47 & $-0,47^{\bullet}$ & $-0,20$ & $-0,47^{\bullet}$ \\
V & 0,07 & $0,73^{\bullet}$ & 0,33 & $-0,07$ & $0,73^{*}$ \\
VI & $0,47^{*}$ & $0,87^{\bullet}$ & 0,20 & 0,07 & $-0,07$ \\
VII & 0,33 & $1,00^{\bullet}$ & 0,07 & $-0,07$ & 0,20 \\
VIII & & & & & \\
\hline
\end{tabular}

Ciênc. agrotec., Lavras. V.27, n.2, p.382-388, mar./abr., 2003 
Constatou-se a presença de uma espécie de Diptera: Chloropidae, sendo a mais freqüente, durante os oito estádios de florescimento, quando o número de flores abertas ainda era muito reduzido. A maior freqüência desse inseto foi verificada das 13 às 15 horas, observando-se que os indivíduos coletados apresentavam grãos de pólen aderidos ao seu corpo. Metcalf \& Flint (1984) comentaram que as larvas de muitas espécies dessa família são fitófagas e outras, saprofíticas, embora o adulto alimente-se de néctar. Durante o estádio I de florescimento, observou-se uma baixa densidade populacional das espécies polinizadoras, porém, espécimens de Chloropidae encontraram a fonte alimentar disponível e estiveram presentes durante todo o período avaliado, com um pico coincidente com o estádio III de florescimento, pois, além de néctar, o número de flores em antese foi grande, encontrando uma massa polínica disponível nas anteras (Figura 1).

Houve uma correlação positiva entre a ocorrência dessa espécie e a umidade relativa nas amostragens efetuadas às 11 horas e 13 horas $(r=0,57$ e $r=0,43$, respectivamente). Provavelmente esse fator ambiental favoreceu a presença desses insetos, facilitando a busca de alimento (Tabela 1).

Nos estádios III e IV, o número de flores em antese foi elevado e, portanto, esperava-se encontrar um grande número de insetos polinizadores, considerando que as condições ambientais fossem favoráveis. No entanto, constatou-se uma redução da densidade populacional do estádio III para o estádio IV devido, provavelmente, ao abaixamento da temperatura, que foi de $16,1^{\circ} \mathrm{C}$ (Figura 1). Verificou-se uma correlação positiva para A. mellifera, B. lecheguana e para uma espécie de Meliponinae com a temperatura durante o estádio III de florescimento (Tabela 2). Durante esses mesmos estádios, a correlação obtida para a umidade relativa com a espécie de Diptera: Chloropidae e os meliponíneos foi negativa, significando que, provavelmente, esse foi um fator importante na redução da população desse inseto.

Nos estádios V e VI de florescimento, durante a senescência, observou-se um segundo pico populacional de insetos, provavelmente devido às condições ambientais favoráveis. Após o estádio V, houve redução no número de flores abertas, e após a senescência, iniciou-se a maturação dos frutos, acarretando uma diminuição das populações de insetos (Figura 1). A população de Trigona sp., no horário de 9 as 11 horas, diminuiu (Figura 1) e, nesse período, a temperatura média foi de $33^{\circ}$ e $34^{\circ} \mathrm{C}$, respectivamente. A análise de correlação entre o número de indivíduos de
Trigona sp. e o horário das 9 e 11 horas significou que a temperatura foi provavelmente o fator que favoreceu a redução populacional (Tabela 1). A correlação observada nos estádios VI, VII e VIII e a umidade relativa (Tabela 2) indicou que esse fator influenciou a redução populacional de Trigona sp., associada ao número de horas de brilho solar na amostragem das 13 horas (Tabela 1).

Nos estádios VII e VIII de florescimento, quando o número médio de indivíduos de A. mellifera e Trigona sp. reduziu, $B$. lecheguana e os Meliponinae passaram a explorar o hábitat, competindo entre si e com os cloropídeos que, nesse estádio, encontravam-se em pequeno número. Os indivíduos da subfamília Meliponinae ocorreram no horário das 7 às $17 \mathrm{~h}$, em densidade populacional inferior, quando comparado com as outras espécies. A correlação apresentada na Tabela 1 evidenciou que a temperatura e a umidade relativa favoreceram sua ocorrência.

A variação na temperatura, umidade relativa e horas de insolação afetou o número de abelhas (Tabela 1). Williams (1985) observou que a concentração de açúcar no néctar foi influenciada pela umidade relativa e horário, constatando-se, ainda, que a densidade de abelhas aumentou com a temperatura, sendo maior durante a floração. $\mathrm{O}$ autor citado acima observou que a maior população de polinizadores ocorreu quando havia um grande número de flores em antese e condições ambientais favoráveis, concordando com os resultados obtidos na pesquisa.

\section{CONCLUSÕES}

Os insetos mais freqüentes nas inflorescências de Brassica napus foram os dípteros da família Chloropidae, as abelhas Trigona sp. e Apis mellifera e o vespídeo Brachygastra lecheguana, como possíveis polinizadores.

Chloropidae Trigona sp., Apis mellifera, Brachygastra lecheguana apresentaram maior frequiência durante o estádio III de florescimento e no período das 9 às 15 horas, com o pico populacional das 13 às 15 horas. 11 horas.

O pico populacional de Trigona sp. foi das 9 às

A variação na temperatura, umidade relativa e horas de insolação afetou o número de abelhas. Para Trigona sp., a temperatura foi um fator que interferiu nas atividades de busca de alimento. 


\section{AGRADECIMENTOS}

À CAPES, pelo suporte financeiro, e aos acadêmicos Cleber Popov e Célia Vasconcelos, do Centro Universitário da Grande Dourados (UNIGRAN), pela valiosa colaboração na coleta e triagem dos insetos em laboratório.

\section{REFERÊNCIAS BIBLIOGRÁFICAS}

BUTIGNOL, C. A. Ocorrência de insetos em capítulos de girassol em distintos horários e estádios de florescimento. Anais da Sociedade Entomológica do Brasil, Jaboticabal, v. 19, n. 2, p. 273-280, 1990.

FREE, J. B. Insect pollination of crops. London: Academic, 1970, $544 \mathrm{p}$.

INFORMATIONS TECHNIQUES CETIOM. Cahiers techniques-insects et autres ravageur. Stades repères du colza. [S.1.], 1985. p. 4.

JOHNSON, L. K.; HUBELL, S. P. Agression and competition among stingless bees: field studies. Ecology, Durham, v. 55, n. 1, p. 120-127, 1974.

KEVAN, P. G.; EISIKOWITCH, D. The effects of insect pollination on canola (Brassica napus L. cv.O.A.C. Triton) seed germinaton. Euphytica, Wageningen, n. 45, p. 39-41, 1990.

KNUTH, P. Handbook of flower pollination. Oxford: Oxford University, 1908. 639 p.

MARTIN, N. B.; NOGUEIRA JÚNIOR, S. Canola: uma alternativa agrícola de inverno para o centro-sul brasileiro. Informações Econômicas, São Paulo, v. 23, n. 4, p. 9-23, 1993.

METCALF, C. L.; FLINT, W. P. Insectos destructivos e insectos utiles: sus costumbres y su control. México: Continental, 1984. 1206 p.
MOHAMMED, A. Pollination studies in Toria (Brassica napus L. var. dichotoma Prain) and Sarson (Brassica campestris L. var. sarson, Prain). Indian. Journal of Agricultural Science, Cambridge, v. 5, p. 125-154, 1935.

MUSSURY, R. M.; FERNANDES, W. D. Studies of the floral biology and reproductive system of Brassica napus L. (Cruciferae). Brazilian Archives of Biology and Technology, Curitiba, v. 43, n. 1, p. 111-17, 2000.

PAWLIKOWSKI, T. Food relationships of Apoidea occurring on winter rape crops and other plants in the neighbourhood of Torum (Poland). Polskie Pismo Entomologiczne, Warszawa, v. 48, p. 267-277, 1978.

SANTOS, B. Flutuação populacional de Plutella xylostella L. em canola (B. napus var. oleifera). In: CONGRESSO BRASILEIRO DE ENTOMOLOGIA, 15., 1995, Caxambú. Anais... Lavras: SEB/ESAL, 1995. p. 182.

SAZIMA, I.; SAZIMA, M. Mamangavas e irapuás (Hymenoptera, Apoidea): visitas, interações e consequiências para polinização do maracujá (Passifloraceae). Revista Brasileira de Entomologia, São Paulo, v. 33, n. 1, p. 109-118, 1989.

SNEDECOR, G. W.; COCHRAN, G. W. Statistical methods. Iowa: State University, 1989. 491 p.

WILLIAMS, I. H. The pollination requirements of swede rape (Brassica napus L.) and of turnip rape (Brassica campestris L.). Journal of Agricultural Science, Cambridge, v. 91, p. 343-348, 1978.

WILLIAMS, I. H. The polinnization of swede rape (Brassica napus L.). Bee World, Bucks, v. 66, n. 1, p. 16-20, 1985. 